\title{
ARTICLE \\ Cryo-EM structure of human mTOR complex 2
}

\author{
Xizi Chen ${ }^{1,2,3}$, Mengjie Liu ${ }^{1,2,3}$, Yuan Tian ${ }^{1,2,3}$, Jiabei Li ${ }^{1,2,3}$, Yilun Qi ${ }^{1,2,3}$, Dan Zhao ${ }^{1,2,3}$, Zihan Wu $u^{1,2,3}$, Min Huang ${ }^{4}$, \\ Catherine C. L. Wong ${ }^{4,5,6}$, Hong-Wei Wang ${ }^{7}$, Jiawei Wang ${ }^{7}$, Huirong Yang ${ }^{1,2,3}$ and Yanhui $\mathrm{Xu}^{1,2,3,8}$
}

\begin{abstract}
Mechanistic target of rapamycin (mTOR) complex 2 (mTORC2) plays an essential role in regulating cell proliferation through phosphorylating AGC protein kinase family members, including AKT, PKC and SGK1. The functional core complex consists of mTOR, $\mathrm{mLST}$, and two mTORC2-specific components, Rictor and mSin1. Here we investigated the intermolecular interactions within mTORC2 complex and determined its cryo-electron microscopy structure at $4.9 \AA$ resolution. The structure reveals a hollow rhombohedral fold with a 2-fold symmetry. The dimerized mTOR serves as a scaffold for the complex assembly. The N-terminal half of Rictor is composed of helical repeat clusters and binds to mTOR through multiple contacts. mSin 1 is located close to the FRB domain and catalytic cavity of mTOR. Rictor and $\mathrm{mSin} 1$ together generate steric hindrance to inhibit binding of FKBP12-rapamycin to $\mathrm{mTOR}$, revealing the mechanism for rapamycin insensitivity of mTORC2. The mTOR dimer in mTORC2 shows more compact conformation than that of mTORC1 (rapamycin sensitive), which might result from the interaction between mTOR and Rictor-mSin 1. Structural comparison shows that binding of Rictor and Raptor (mTORC1-specific component) to mTOR is mutually exclusive. Our study provides a basis for understanding the assembly of $\mathrm{mTORC2}$ and a framework to further characterize the regulatory mechanism of mTORC2 pathway.
\end{abstract}

Cell Research (2018) 28:518-528; https://doi.org/10.1038/s41422-018-0029-3

\section{INTRODUCTION}

Mechanistic target of rapamycin (mTOR) is a giant Ser/Thr kinase and is evolutionarily conserved in eukaryotes from yeast to human. ${ }^{1,2}$ mTOR functions as a master regulator in controlling various cellular processes through forming two distinct multisubunit protein complexes, a rapamycin-sensitive mTOR complex 1 (mTORC1) and a rapamycin-insensitive mTOR complex 2 (mTORC2). ${ }^{3-6}$ Upon stimulation by growth factors, energy and amino acids, mTORC1 controls cell growth through regulating signaling pathways of translation, transcription, nutrient uptake, ribosome biogenesis and autophagy. In contrast, mTORC2 primarily functions as an effector of insulin-PI3K (phosphatidylinositide 3-kinases) pathway and regulates cell proliferation. The representative mTORC2 substrates are AGC (PKA/PKG/PKC) protein kinase family members, including $\mathrm{AKT}^{7} \mathrm{PKC}^{8}$ and SGK1. ${ }^{9}$ Aberrant mTORC2 signaling has been shown to be involved in tumorigenesis and $\mathrm{mTORC2}$ may serve as a potential anti-cancer drug target. ${ }^{10}$

The mTORC1 and mTORC2 complexes share two core components, mTOR and mammalian lethal with SEC13 protein 8 (mLST8, also known as GBL). ${ }^{11}$ Regulatory-associated protein of mTOR (Raptor) is the mTORC1-specific component. ${ }^{12,13}$ mTORC2 contains two essential specific components, rapamycin insensitive companion of mTOR (Rictor) ${ }^{8,14}$ and mammalian stress-activated protein kinase-interaction protein 1 (mSin1)(Fig. 1a). ${ }^{15,16}$ Yeast TORC2 is composed of four essential components, Tor2 (mTOR ortholog), Lst8 (mLST8 ortholog), Avo1 (mSin1 ortholog), Avo3 (Rictor ortholog), and two non-essential components, Avo2 (no human ortholog) and Bit61/2 (Protor $1 / 2$ equivalent). ${ }^{6}$

Eukaryotic mTOR is a protein kinase belonging to the family of PI3K-related kinases (PIKK). ${ }^{17,18}$ Representative PIKK family members include three master regulators for DNA damage responses, DNA-dependent protein kinase catalytic subunit (DNA-PKcs), ataxia-telangiectasia mutated (ATM), and ATM- and Rad3-related (ATR). The PIKK family members share conserved domain architecture, a large N-terminal a-solenoid, a FAT (FRAP, ATM, TRRAP) domain, a kinase domain (KD) with a FKBP12 (12 kDa FK506-binding protein)-rapamycin binding (FRB) insert (Fig. 1b). Rapamycin inhibits mTORC1 activity by forming a complex with immunophilin FKBP12. ${ }^{3,8}$ FKBP12 and rapamycin together bind to FRB, prohibit substrate entry and therefore inhibit mTORC1 kinase activity. ${ }^{19}$ The $\mathrm{PH}$ domain of $\mathrm{mSin} 1$ inhibits mTORC2 activity and this autoinhibition is released in the presence of phosphatidylinositol $(3,4,5)$-trisphosphate (PIP3). ${ }^{20}$ It remains elusive why mTORC2 is insensitive to FKBP12-rapamycin and how the $\mathrm{PH}$ domain inhibits mTORC2 activity.

\footnotetext{
${ }^{1}$ Fudan University Shanghai Cancer Center, Institutes of Biomedical Sciences, Shanghai Medical College of Fudan University, Shanghai 200032, China; ${ }^{2}$ Key Laboratory of

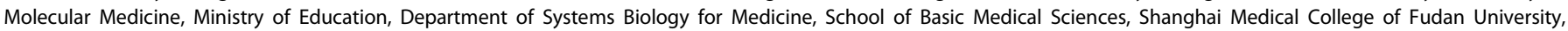

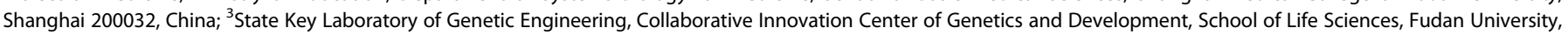

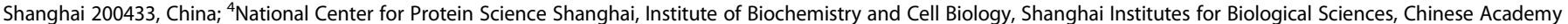

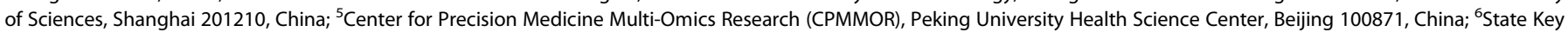
Laboratory of Natural and Biomimetic Drugs, School of Pharmaceutical Science, Peking University, Beijing 100871, China; ${ }^{7}$ Ministry of Education Key Laboratory of Protein

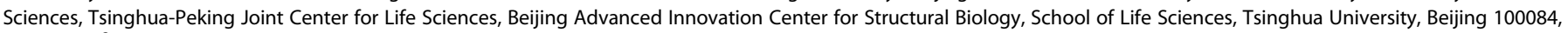
China and ${ }^{8}$ CAS Center for Excellence in Molecular Cell Science, Chinese Academy of Sciences, Shanghai 200031, China

Correspondence: Huirong Yang (yanghr@fudan.edu.cn) or Yanhui Xu (xuyh@fudan.edu.cn)

These authors contributed equally: Xizi Chen, Mengjie Liu In PDF, please change running title to "Cryo-EM structure of human mTORC2"
}

Received: 6 January 2018 Revised: 2 February 2018 Accepted: 24 February 2018

Published online: 22 March 2018 
a

$1 \quad 296$

592

888

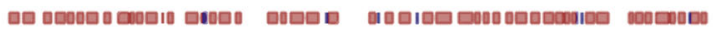
1184 ARM

Rictor

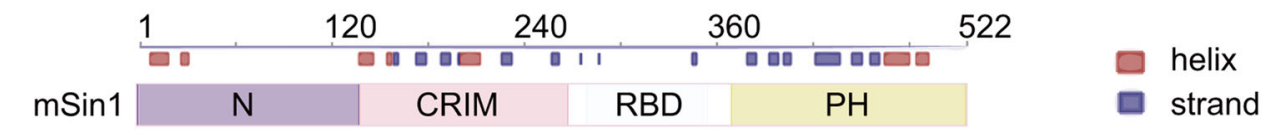

b

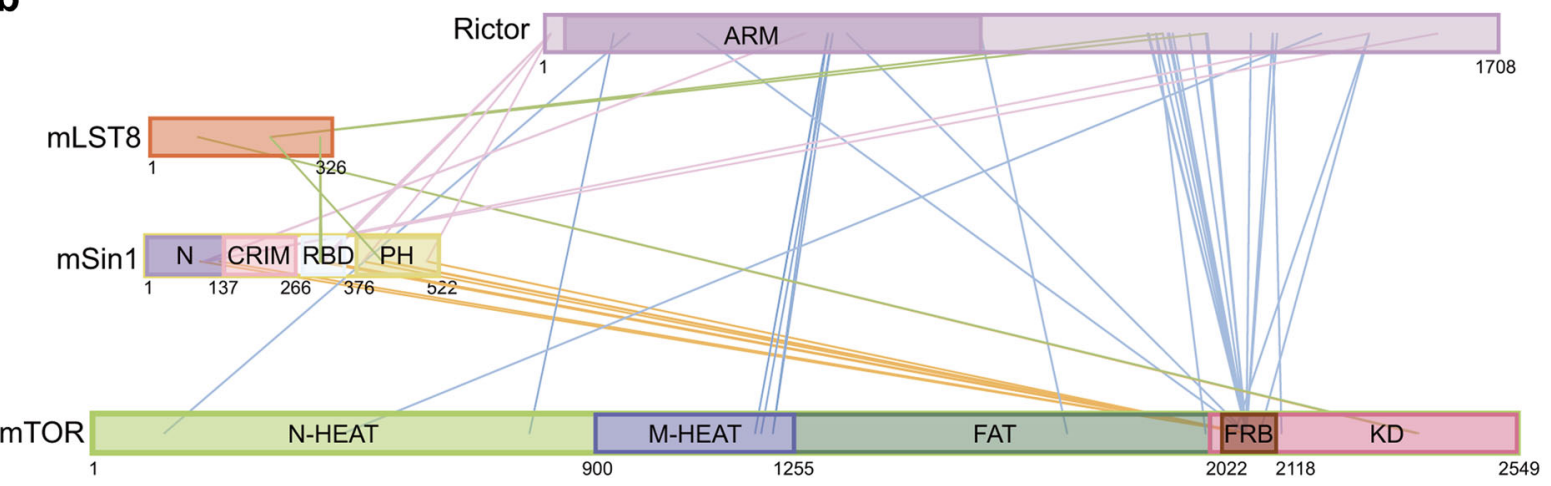

Fig. 1 Cross-linking mass spectrometry (XL-MS) analysis of mTORC2 complex. a Secondary structure prediction of Rictor and mSin1, the two mTORC2-specific components. Predicted helices and strands are indicated by red and blue squares, respectively. The N-terminal portion of Rictor is predicted to be armadillo (ARM) repeat clusters. b Schematic representation of the intermolecular cross-links within mTORC2 complex. The identified intermolecular cross-links are indicated by color-coded solid lines. The intramolecular cross-links were omitted for simplicity. N-HEAT, N-terminal HEAT repeats; M-HEAT, middle HEAT repeats; FAT, FRAP, ATM, TRRAP domain; KD, kinase domain; FRB, FKBP12rapamycin binding; $\mathrm{N}$, N-terminal region of $\mathrm{mSin} 1$; $\mathrm{CRIM}$, conserved region in the middle; $\mathrm{RBD}$, Ras binding domain; $\mathrm{PH}$, pleckstrin homology

The three-dimensional structure of mTORC 1 has been extensively studied in the past years. ${ }^{19,21-23}$ Recently, Yang et al. ${ }^{24}$ reported atomic resolution structure of mTORC1 and related complexes and revealed the mechanism for mTORC1 activation by Ras homologue enriched in brain (Rheb) and inhibition by prolinerich Akt/PKB substrate $40 \mathrm{kDa}$ (PRAS40). Negative stain $(\sim 26 \AA)^{25}$ and cryo-electron microscopy (EM) $(7.9 \AA)^{26}$ structures of S. cerevisiae TORC2 (scTORC2) reveal an overall fold and subunit architecture of the complex. ${ }^{27}$ However, the structure of mammal/ human mTORC2 remains largely unknown. Here we report the cryo-EM structure of human mTORC2 at $4.9 \AA$ resolution. The structural and biochemical analyses together provide a structural model for understanding the assembly and function of mTORC2 complex.

\section{RESULTS}

Purification and cross-linking mass spectrometry analysis of mTORC2

To perform biochemical and structural analyses of mTORC2, we purified human mTORC2 complex to homogeneity (Supplementary information, Figure S1a). The four mTORC2 components were co-transfected into HEK293F cells in suspension culture and the purified complex consists of mTOR, Rictor, mSin 1 and $\mathrm{mLST} 8$. The complex exhibited protein kinase activity in the in vitro kinase assay using purified human AKT (kinase-dead mutant) as substrate and the kinase activity was inhibited by Torin1, a wellcharacterized ATP-competitive inhibitor of mTOR (Supplementary information, Figure S1b).

Secondary structure prediction and previous study show that $\mathrm{mSin} 1$ is composed of an N-terminal region (residues 1-137, designated $m \operatorname{Sin} 1^{\mathrm{N}}$ ), a conserved region in the middle (CRIM, 138-266), a Ras binding domain (RBD, 279-353), and a pleckstrin homology domain $(\mathrm{PH}, 376-522)^{20}$ (Fig. 1a). Rictor has an $\mathrm{N}$ terminal armadillo (ARM) repeat cluster ( 900 residues), followed by a large unstructured region. ${ }^{28}$ We next performed cross-linking mass spectrometry (XL-MS) analysis using the purified mTORC2 complex (Fig. $1 \mathrm{~b}$ and Supplementary information, Table S1). The major intermolecular cross-links identified are summarized below. (1) The FRB domain of mTOR makes multiple contacts with $\mathrm{mSin} 1$ and C-terminal regions (residues 1,100-1,500) of Rictor. (2) The C-terminal portion (residues 1,186-1,218) of MHEAT of mTOR makes contacts with the N-terminal portion (residues 506-516) of Rictor. (3) mSin 1 makes multiple contacts with Rictor, mainly with its $\mathrm{N}$-terminal region. Compared to the $\mathrm{XL}$ MS analysis of scTORC2 complex, ${ }^{25}$ more cross-links were observed between the FRB and the two mTORC2-specific components, Rictor and mSin1. This is consistent with our structural analysis (discussed below).

\section{Rictor-mTOR interaction}

We next performed in vitro immunoprecipitation assays to investigate the intermolecular interaction of the four mTORC2 components. The maltose-binding protein (MBP)-tagged Rictor could pull out the endogenous mTOR in the presence of $\mathrm{mSin} 1$ (Supplementary information, Figure S2a, lanes 3 and 7) and the overexpressed mTOR in the absence of $\mathrm{mSin} 1$ (lanes 2 and 6). More stable Rictor-mTOR interaction could be detected in the presence of both $\mathrm{mSin} 1$ and $\mathrm{mLST} 8$ (lane 8 ), suggesting that $\mathrm{mSin} 1$ and $\mathrm{mLST} 8$ may facilitate the interaction between Rictor and mTOR. Two C-terminal truncations and an N-terminal truncation of Rictor could bind to mTOR (Fig. 2a). These observations are consistent with the XL-MS result (Fig. 1b), and suggests multiple contacts between Rictor and mTOR.

Rictor-mSin 1 interaction

MBP-tagged Rictor bound to $\mathrm{mSin} 1$ in the absence or the presence of mTOR and/or mLST8 (Supplementary information, Figure S2a, lanes 3, 5, 7, and 8), indicating a direct interaction independent of mTOR-mLST8. Consistently, the C-terminally protein $\mathrm{A}$ (ProA)-tagged $\mathrm{mSin} 1$ directly bound to Rictor (Supplementary information, Figure S2b, lane 3). 
a

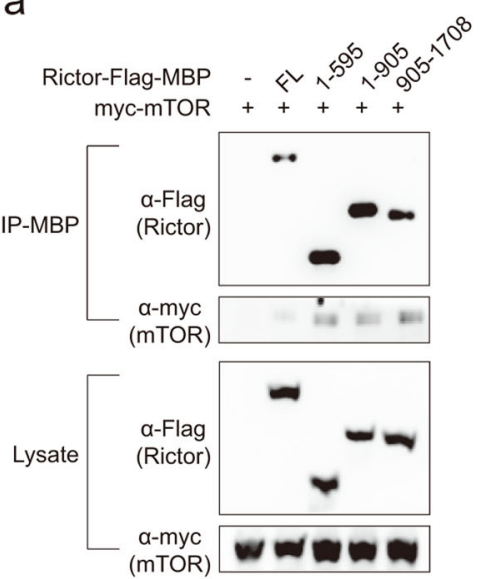

C
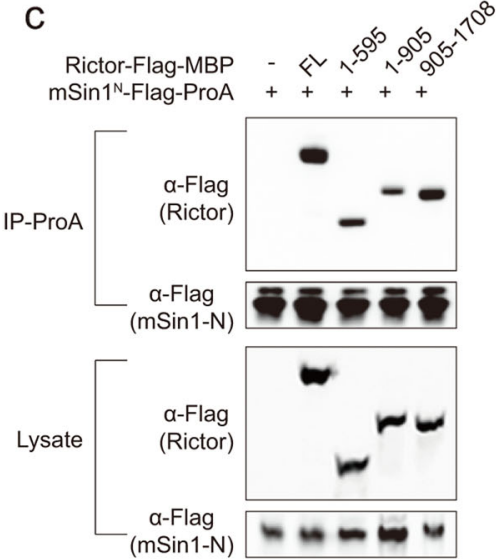

b

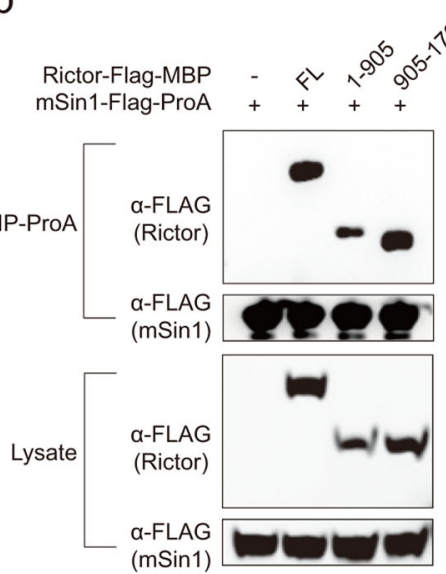

d
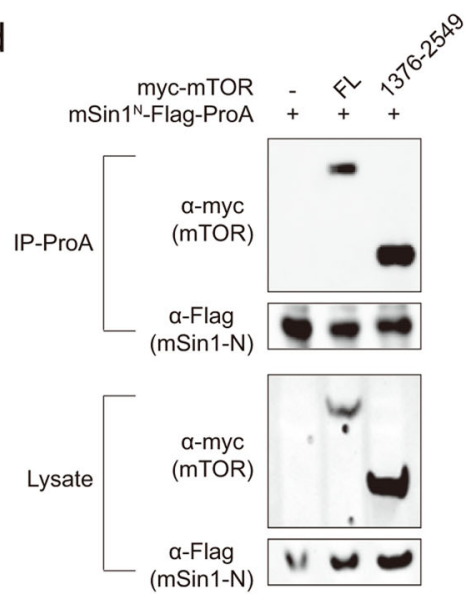

e
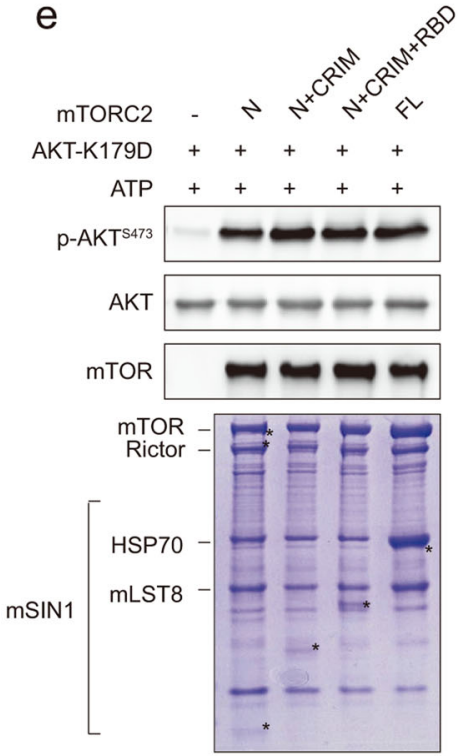

Fig. 2 Intermolecular interactions within mTORC2 complex. a Co-immunoprecipitations of full-length mTOR and various Rictor truncations. Various Rictor truncations and myc-tagged mTOR were co-transfected into 293F cells and Rictor proteins (containing C-terminal Flag and MBP tags) were immobilized using Amylose resins. The bound proteins and whole cell lysates were subjected to SDS-PAGE and visualized by western blotting using antibodies as indicated. b, c Co-immunoprecipitations of various Rictor truncations and full-length $\mathrm{mSin} 1$ (b) or $\mathrm{mSin} 1 \mathrm{~N}$ (c). d Co-immunoprecipitations of $\mathrm{mSin} 1^{\mathrm{N}}$ with full-length and truncated $\mathrm{mTOR}$. The experiments were performed as described in a. e In vitro kinase assay using purified mTORC2 containing full-length or truncated mSin1. Purified human AKT (K179D, kinase-dead mutant) serves as a substrate. The activities of mTORC2 were detected by immunoblotting with antibodies targeting phospho-Ser473 of AKT

Immunoprecipitation assays show that $\mathrm{mSin} 1^{\mathrm{N}}$-containing constructs of $m \operatorname{Sin} 1$, including $m \operatorname{Sin} 1^{\mathrm{N}}, \mathrm{mSin} 1^{\mathrm{N}+\mathrm{CRIM}}$ and $\mathrm{mSin} 1^{\mathrm{N}+\mathrm{CRIM}+\mathrm{RBD}}$, bound to Rictor with a binding affinity comparable to that of full-length $\mathrm{mSin} 1$ (Supplementary information, Figure S3a, lanes $1-4$ ). In contrast, constructs lacking $\mathrm{mSin} 1^{\mathrm{N}}$ all showed undetectable interaction with Rictor (lanes 5-10). The results indicate a critical role of $m \operatorname{Sin}^{\mathrm{N}}$ in mediating $\mathrm{mSin} 1$-Rictor interaction. Full-length $\mathrm{mSin} 1$ bound to the $\mathrm{N}$-terminal portion (residues 1-905) or C-terminal portion (residues 905-1708) of Rictor (Fig. 2b). Moreover, $\mathrm{mSin} 1^{\mathrm{N}}$ could bind to the Rictor $\mathrm{N}-$ terminal portions containing residues $1-595$ or $1-905$ and $\mathrm{C}-$ terminal portion containing residues 905-1708 (Fig. 2c). Taken together, $\mathrm{mSin} 1$ and Rictor make multiple contacts, and $m \operatorname{Sin} 1^{\mathrm{N}}$ is critical for the intermolecular interaction.

mSin1-mTOR interaction

The ProA-tagged $\mathrm{mSin} 1$ could pull out endogenous mTOR, indicating a direct interaction (Supplementary information, Figure S2b, lanes 1, 3, 4, and 7). Full-length $\mathrm{mSin} 1$ and two Cterminal truncations could also pull out the endogenous mTOR in the presence of Rictor (Supplementary information, Figure S3a, lanes $1,3,4)$. The endogenous mTOR may compete with the overexpressed mTOR (myc-mTOR), leading to a relatively weak interaction between myc-mTOR and mSin1 (Supplementary information, Figure S2b, lane 2). The interaction between $\mathrm{mSin} 1$ and myc-mTOR was moderately enhanced in the presence of Rictor (lane 5) or mLST8 (lane 6), and largely enhanced in the presence of both Rictor and $\mathrm{mLST} 8$ (lane 8 ). mSin 1 bound to mLST8 (lane 4) and the interaction was enhanced when mTOR was overexpressed (lane 6).

The $\mathrm{mSin} 1^{\mathrm{N}}$-containing truncations of $\mathrm{mSin} 1$ bound to the overexpressed mTOR with relatively weaker binding affinity compared to that of the full-length mSin1 (Supplementary information, Figure $\mathrm{S} 3 \mathrm{~b}$, lanes $1-4)$. The deletion of $\mathrm{mSin} 1^{\mathrm{N}}$ largely decreased the mSin $1-m T O R$ interaction (Supplementary information, Figure $\mathrm{S} 3 \mathrm{~b}$, lanes $5-10)$. The $\mathrm{mSin} 1^{\mathrm{N}}{ }_{\text {-containing }}$ truncations of $\mathrm{mSin} 1$ could pull out other three mTORC2 components and form the whole mTORC2 complex (Supplementary information, Figure S3c, lanes 1-4). In contrast, the constructs with deletion of $\mathrm{mSin} 1^{\mathrm{N}}$ could not pull out any of the three components (Supplementary information, Figure S3c, lane 5-10). Moreover, $m \sin 1^{\mathrm{N}}$ directly bound to full-length $\mathrm{mTOR}$ and $\mathrm{C}$ terminal portion of mTOR (residues 1,376-2,549) (Fig. 2d). The $\mathrm{mSin} 1^{\mathrm{N}}$-containing constructs could form stable and catalytically active mTORC2 complexes (Fig. 2e). Taken together, mSin1 has multiple contacts with other mTORC2 components and $\mathrm{mSin} 1^{\mathrm{N}}$ plays a critical role in mediating the $\mathrm{MTORC2}$ complex formation. 

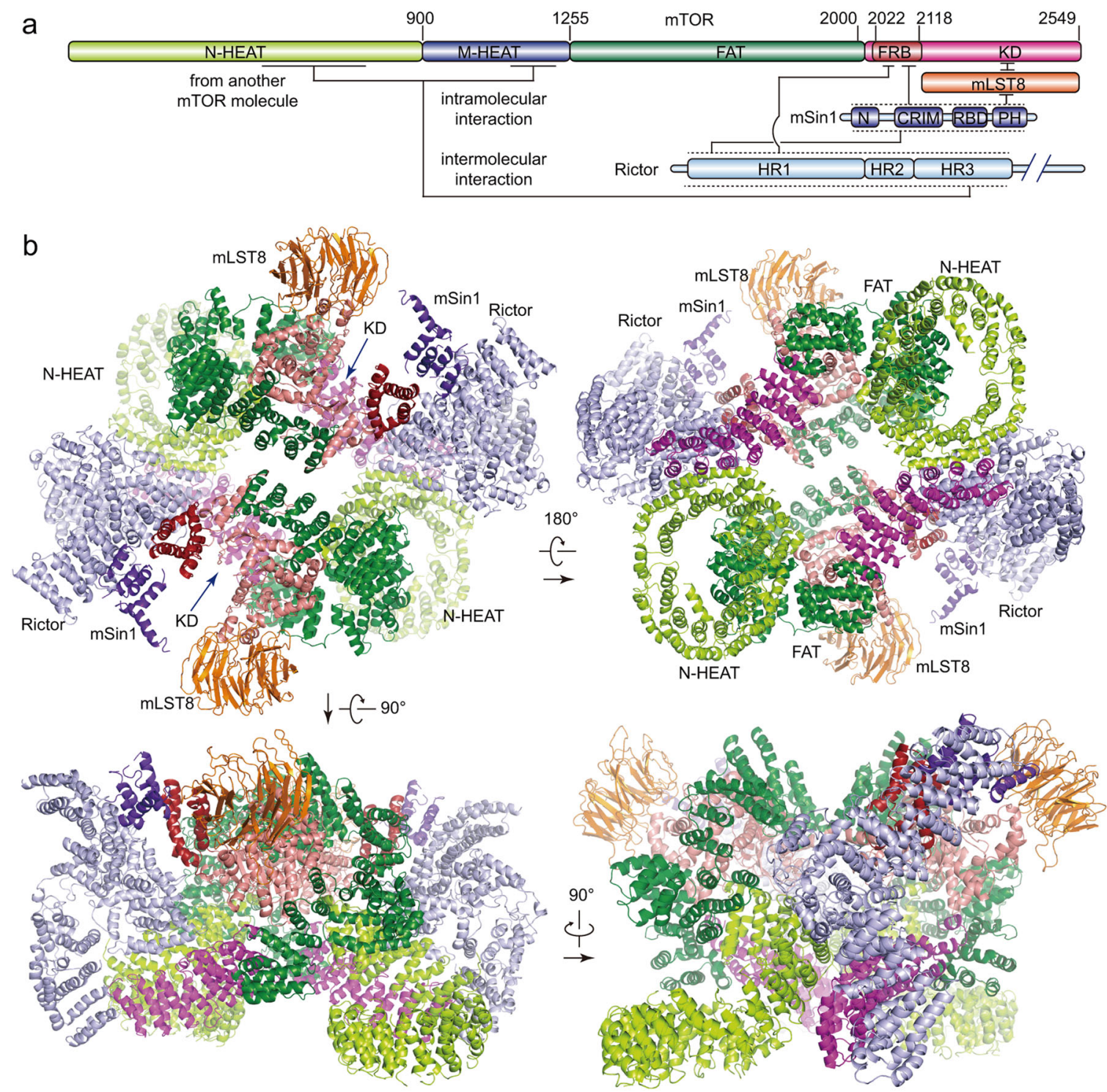

Fig. 3 Overall structure of human mTORC2 complex. a Color-coded domain architecture of the four human mTORC2 components, mTOR, Rictor, $\mathrm{mSin} 1$ and $\mathrm{mLST} 8$. The same color scheme is used hereafter in all structure figures if not otherwise specified. The inter- and intramolecular contacts are shown as connected solid lines. The dashed lines represent unassigned regions of Rictor and mSin 1 that are involved in intermolecular contacts. b Ribbon representations of the mTORC2 complex in four different views with domains indicated

This is consistent with our XL-MS analysis and previous studies. $^{15,16}$

\section{Cryo-EM structure determination}

We next determined the cryo-EM structure of mTORC2 using single-particle reconstruction and the EM map was refined to $4.9 \AA$ resolution (Supplementary information, Figure S4 and Table S2). The cryo-EM map shows well-defined secondary structural elements (Supplementary information, Figure S5). The models of mTOR and mLST8 were built according to the EM map and structural models of mTORC1. ${ }^{23,24}$ However, the models of the Rictor and $\mathrm{mSin} 1$ could not be unambiguously defined merely from the EM map.

We next built structural models of Rictor and $\mathrm{mSin} 1$ based on the EM map, the secondary structure prediction (Fig. 1a), the XLMS (Fig. 1b), and the in vitro immunoprecipitation assays (Fig. 2; Supplementary information, Fig. S2 and Fig. S3). Apart from the density corresponding to mTOR-mLST8, extra EM density should be derived from Rictor and/or mSin1 (Supplementary information, Figure S5). The EM map reveals primarily helical elements that can accommodate $\sim 1,000$ residues in total for each mTORC2 protomer. Secondary structure prediction indicates that the helical elements are likely to be derived mainly from the $\mathrm{N}$-terminal portion of Rictor, which is composed of ARM and/or HEAT repeat domain clusters. ${ }^{28}$ We therefore built model of Rictor as polyAlanine chains (880 residues) into this EM density (Supplementary information, Figure S5c and e).

The EM map shows that a four-helix bundle is close to the FRB domain and the catalytic cavity of mTOR and a helical region bridges $\mathrm{mLST} 8$ and Rictor/mSin1 (Supplementary information, Figure $\mathrm{S} 5 \mathrm{c}$ and d). Biochemical analyses show that $\mathrm{mSin} 1^{\mathrm{N}}$ directly binds to the N-terminus (1-595) of Rictor and XL-MS analysis shows that multiple regions of $\mathrm{mSin} 1$ are close to the FRB domain of mTOR. Therefore, these five a-helices are very likely to be derived from $\mathrm{mSin} 1$. We built $\mathrm{mSin} 1$ model as five isolated polyAlanine chains into the EM map. The C-terminal half of Rictor and most of the mSin 1 portion are invisible in the EM map, which might result from their intrinsic flexibility. 
The overall structure of mTORC2

The mTORC2 structure reveals a hollow rhombohedral fold with overall dimensions of $\sim 220 \times 200 \times 130\left(\AA^{3}\right)$ (Fig. 3). The complex adopts a 2-fold symmetry and each protomer consists of one copy of mTOR, mSin1, Rictor and mLST8. Dimer of mTOR-mLST8 heterodimer in mTORC2 adopts a similar overall architecture to that in mTORC1 with a root mean square deviation (RMSD) of $6.7 \AA$ for 3,550 $\mathrm{Ca}$ atoms (Supplementary information, Figure S6a). As observed in the mTORC1, the two mTOR monomers of mTORC2 pack against each other and form a central scaffold to provide binding surfaces for the other three components. The two copies for each individual mTORC2 subunits (mLST8, mSin1 or Rictor) symmetrically bind to the mTOR dimer (Fig. 3b). Consistent with previous studies, $\mathrm{mLST} 8$ stably binds to the kinase domain of mTOR and flanks outside of the core complex. ${ }^{19,21,23}$

As shown in previous studies, mTOR is composed of an $\mathrm{N}$ terminal super-helical HEAT repeat (N-HEAT), a middle extended HEAT repeat (M-HEAT), a "C"-shaped a-solenoid (FAT), followed by the kinase domain with a FRB insertion (Supplementary information, Figure S6b). The XL-MS analysis and high-resolution cryo-EM structure of mTORC $1^{24}$ strongly support that mTOR adopts a topological conformation as described in previous studies of mTORC $1^{23}$ and Tor-Lst8 from the thermotolerant yeast Kluyveromyces marxianus ${ }^{22}$ (Supplementary information, Figure S6b).

\section{Rictor and Rictor-mTOR interface}

Rictor has three continuous ARM/HEAT helical repeat (HR) clusters, HR1 to HR3. HR1 is composed of nine parallel helical repeats and adopts an extended conformation (Fig. 4). HR2 is composed of eight a-helices and binds to the repeats 5-9 of HR1 in a ridge-toridge manner. HR3 adopts an extended conformation and has six helical repeats. HR3 has no direct contact with HR1 or HR2 and its conformation is stabilized through interacting with mTOR (discussed below).

We observed three major intermolecular contacts between Rictor and mTOR (Fig. 5a-c). (1) Helical repeats R1 to R6 of HR1 make contacts with mSin 1 and the FRB domain of mTOR. (2) The three terminal a-helices of HR1 make contacts with the M-HEAT of mTOR and the N-HEAT of the other mTOR molecule (designated N-HEAT'), and thus form a three-way junction for intermolecular interaction. This contact agrees nicely with the XL-MS result, which shows extensive cross-links between Rictor (residues 507-516) and the M-HEAT (residues 1,186-1,218) of mTOR (Fig. 1b). (3) The last two repeats of HR3 make contacts with the last HEAT repeat of the M-HEAT of mTOR. Notably, the XL-MS analysis shows extensive cross-links between the C-terminal portion of Rictor and the FRB of mTOR (Fig. 1b). The C-terminal portion of Rictor could not be assigned in the EM map possibly due to its flexibility. Such RictormTOR contacts suggest a potential regulatory function of Rictor yet to be discovered.

\section{Comparison of mTORC1 and mTORC2 structures}

Although mTORC1 and mTORC2 adopt similar overall conformations, there are two distinct structural features. The first one is their specific components, Rictor and Raptor, which adopt distinct conformations (Fig. 5d). This is consistent with the fact that the two proteins have no sequence homology. Interestingly, Raptor also has an extended HEAT repeat domain, which binds to the MHEAT and the N-HEAT' of mTOR. Raptor and mTOR form a threeway junction in a manner similar to that formed between Rictor and mTOR in mTORC2 (Fig. $5 \mathrm{~d}$ ). mTORC1 has no contacts that are equivalent to the first and third Rictor-mTOR contacts in mTORC2. The structure comparison clearly shows that the binding of Rictor and Raptor to mTOR is mutually exclusive, which agrees nicely with the distinct mTOR complex assemblies. ${ }^{3,8,14}$

Another difference between mTORC1 and mTORC2 is their overall organization of mTOR dimer. As shown in the top view of Fig. 6a, mTOR symmetrically dimerizes through intermolecular

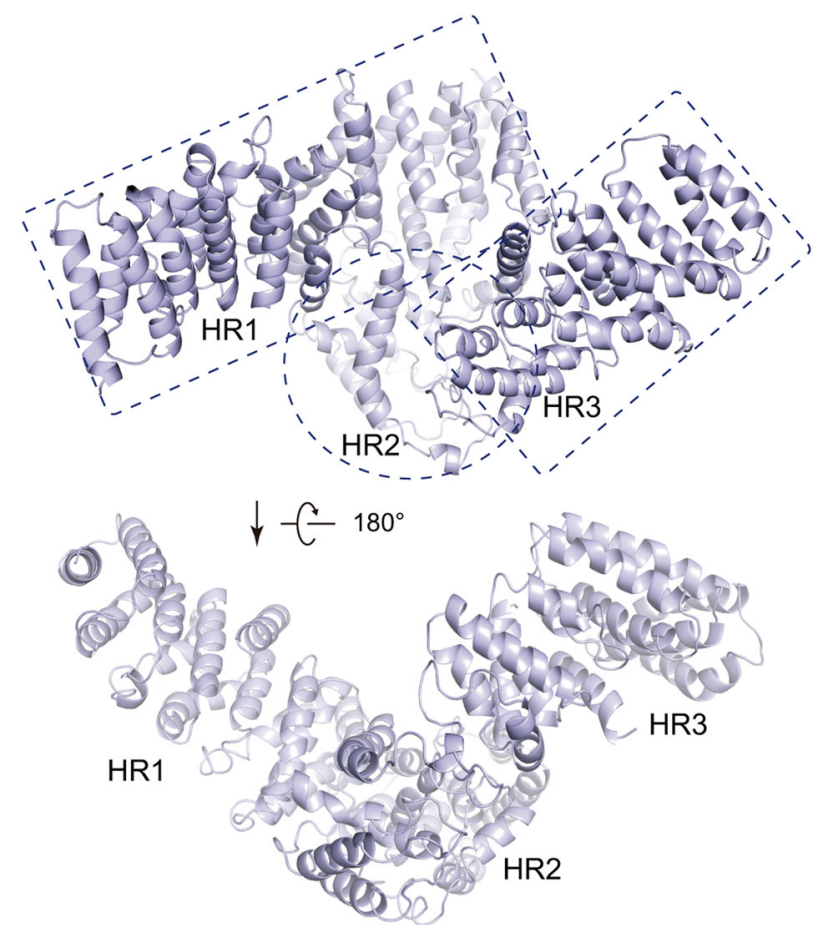

Fig. 4 Structure of Rictor in the mTORC2 complex. Ribbon representations of Rictor in two different views. Three helical ARM/HEAT repeat clusters, HR1 to HR3, are indicated in the dashed squares or circle

interaction between the M-HEAT and N-HEAT'. The patterns for mTOR dimerization are similar in the two complexes. However, as shown in the opposite view, mTORC2 shows a narrower central hole compared to that of mTORC1 (Fig. 6b). The inner height of the hole is represented by the distance between two symmetrically arranged R1966 residues from the two FAT domains. As shown in Fig. $6 c$, the inner hole of mTORC2 is as narrow as $\sim 11 \AA$, whereas that of mTORC1 is $\sim 23 \AA$. Such conformational difference is likely to result from distinct complex assemblies. Compared to the mTORC1 structure, the existence of Rictor and mSin 1 pushes the FRB domain to move forward to the other mTOR by as far as $\sim 12 \AA$ (Fig. $6 \mathrm{~d}$ ). As a result, the FAT and KD domains make a movement to the same direction (Fig. 6b). Therefore, upon association with Rictor and mSin1, mTOR dimer may undergo conformational switch to form a more compact fold than that of mTORC1. Such structural difference of the two complexes may be related to their distinct functional properties.

It is theoretically possible that one Rictor/mSin 1 and one Raptor simultaneously bind to one mTOR dimer because mTORC2 and mTORC1 both have two symmetrically arranged Rictor/Raptor binding surfaces (Fig. 6a, b). However, no such chimera complex has been reported yet. It is tempting to speculate that Rictor/ $\mathrm{mSin} 1$ association might allosterically affect the other binding site to favor Rictor association and prohibit Raptor association. Raptor may have a similar effect on mTOR dimer to inhibit Rictor/mSin1 association.

mSin 1 structure and rapamycin insensitivity

According to the secondary structure prediction and biochemical analyses, we built a structural model for mSin1, which shows a four-helix bundle and an a-helix bridging mLST8 and Rictor/ $\mathrm{mSin} 1$. These helices might be primarily from $\mathrm{mSin} 1^{\mathrm{N}}$ and partly from other $\mathrm{mSin} 1$ regions. Two a-helices pack against helical repeats R1 to R4 of Rictor (Fig. 7a). The bottom of the four-helix bundle and helical repeat R6 of Rictor are close to the FRB of mTOR. Such structural organization agrees nicely with the XL-MS 

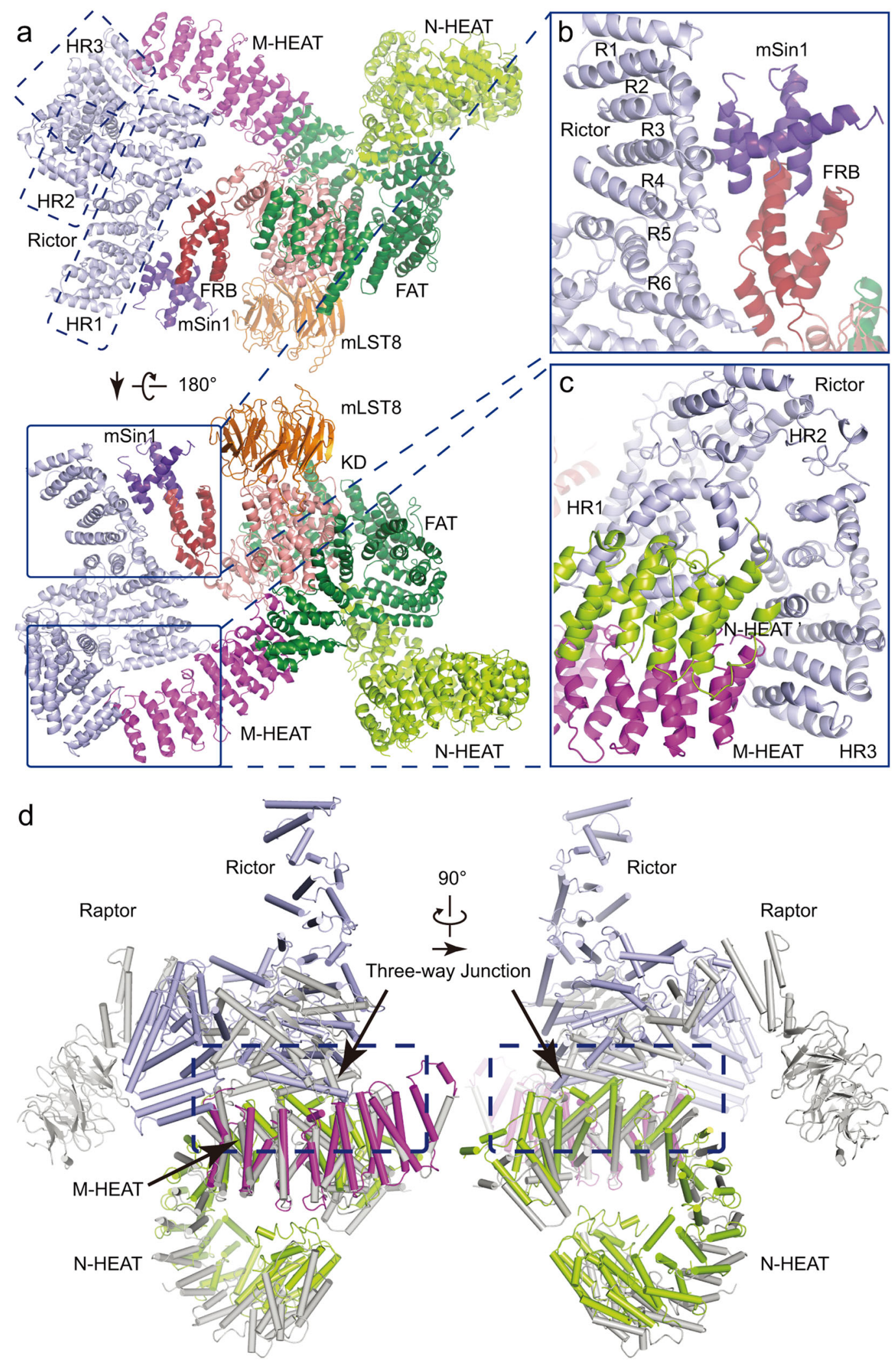

Fig. 5 Intermolecular interactions within mTORC2 complex. a Ribbon representations of mTORC2 protomer in two different views. b, c Closeup views of intermolecular interactions within mTORC2. Ribbon representations are shown in two different views to better illustrate the intermolecular contacts. The three major contacts include one between Rictor and mSin 1 and FRB of mTOR (b), and two between Rictor and mTOR (c). R1-R6 represent the AMR/HEAT helical repeats of Rictor HR1 subdomain. N-HEAT' in c indicates that it is from the other mTOR molecule. The flanking HEAT repeats of this N-HEAT' were not shown for simplicity. d Rictor and Raptor bind to the M-HEAT and N-HEAT' of mTOR and form a three-way junction in mTORC2 and mTORC1, respectively. Superimposition of the mTORC1 (PDB: 5H64) and mTORC2 structures shown in two different views with unnecessary regions omitted. Note that two mTOR molecules are well aligned, whereas Rictor and Raptor adopt distinct conformations. The $\alpha$-helices are shown as cylinders for simplicity 


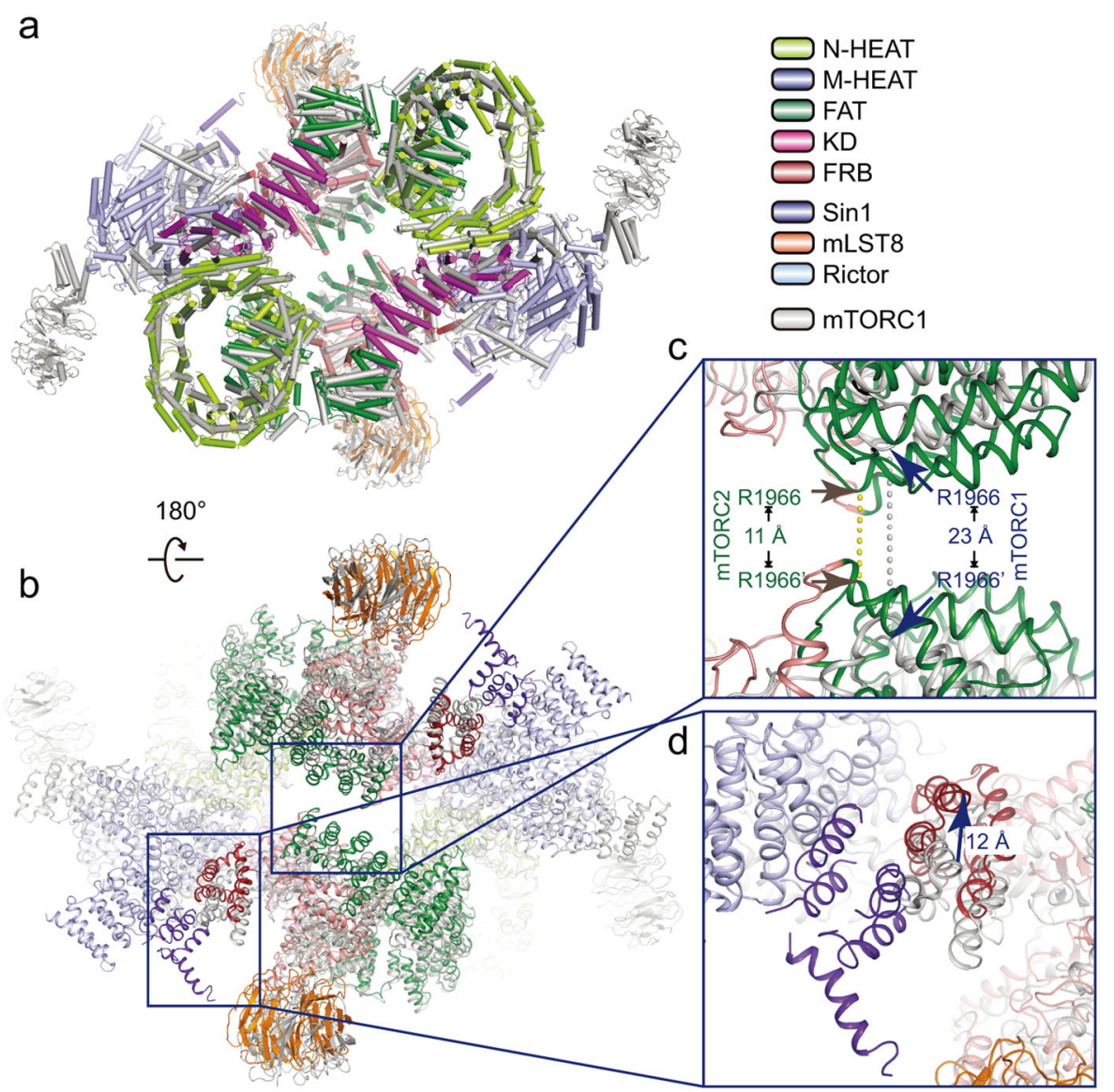

Fig. 6 Structural comparison of mTORC1 and mTORC2. a, b Superimposition of the mTORC1 (PDB: 5H64) and mTORC2 structures shown in two different views. The helices are shown in cylinders (a) and ribbon representations (b), respectively. The color scheme is indicated. $\mathbf{c}$ Closed-up view of the central holes of mTORC1 and mTORC2 for comparison. The distance between residues R1966 and R1966' (from the other mTOR molecule) is indicated for the two complexes. d Closed-up view of the FRB domains of mTORC1 and mTORC2 for comparison. The FRB domain would move upward by as far as $12 \AA$ upon conformational transition from mTORC1 to mTORC2

result, which shows extensive cross-links between $\mathrm{mSin} 1$ and the $\mathrm{N}$-terminus of Rictor, and that between $\mathrm{mSin} 1$ and FRB (Fig. 1b).

FKBP12 and rapamycin together inhibit mTORC1 kinase activity through binding to the FRB domain of mTOR and therefore provide steric hindrance for substrate entry. ${ }^{19}$ In contrast, FKBP12rapamycin does not directly bind mTORC2 or inhibit its kinase activity under physiological conditions. Structural comparison shows that $F R B{ }^{\text {mTORC2 }}$ and the isolated FRB in the structure of FRBrapamycin-FKBP12 (PDB: 1FAP) adopt similar conformations ${ }^{29}$ (Fig. 7a, lower panel). The existence of the five a-helices of $\mathrm{mSin} 1$ would generate steric hindrance and prohibit the association of FKBP12-rapamycin. Therefore, once mTORC2 complex is formed, FKBP12-rapamycin would not be able to get access to the FRB for inhibition. If FKBP12-rapamycin successfully binds to the FRB domain, mSin1-Rictor interaction would not be maintained and the complex will be disrupted. To test this possibility, we performed an in vitro pull-down assay using purified Flagtagged mTORC2 and GST-tagged FKBP12 in the presence of rapamycin (Fig. 7b, c). In the GST pull-down assay, FKBP12 strongly bound to $\mathrm{MTORC} 1$ in the presence of rapamycin but showed no interaction with mTORC2. Note that mTOR was pulled down by FKBP12, which might result from the presence of free mTOR in the mTORC2 sample or the disruption of mTORC2 by FKBP12rapamycin under high protein concentration. Nevertheless, structural analyses clearly show that the mTORC2 complex formation and FRB-FKBP12-rapamycin association are mutually exclusive, which is in line with rapamycin insensitivity of mTORC2.

\section{DISCUSSION}

In this study, we report the cryo-EM structure of human mTORC2 at $4.9 \AA$ resolution. The structure provides a molecular basis for understanding the complex assembly of mTORC2 and the regulatory mechanism of $\mathrm{mTORC} 2$. We have used a reasonably large number of particles for structure determination and refinement. The resolution is limited to $4.9 \AA$ resolution, which might result from the intrinsic dynamic property. Further optimization of sample preparation, such as better cross-linking, may generate well-behaved sample for higher resolution structure determination.

Our biochemical and structural analyses demonstrate that Rictor and $\mathrm{mSin} 1$ interact with each other and together make multiple contacts with mTOR-mLST8. The pattern for complex assembly of mTORC2 is distinct from that of mTORC1, in which Raptor binds to mTOR primarily through one intermolecular contact. Structural comparison shows that the binding of Rictor and Raptor to mTOR is mutually exclusive, and clearly reveals the mechanism for distinct complex assemblies of mTOR. ${ }^{3,8,14}$ Although Rictor and $\mathrm{mSin} 1$ can individually bind to mTOR, both proteins are required for stable mTORC2 complex formation. Lack of Rictor or mSin1 would strongly destabilize mTORC2 complex formation, as judged by chromatography and electron microscopy analyses (data not shown). We were not able to individually purify well-behaved Rictor or mSin 1 proteins (data not shown), suggesting that the two proteins may not be stable if not in the context of mTORC2 complex. 

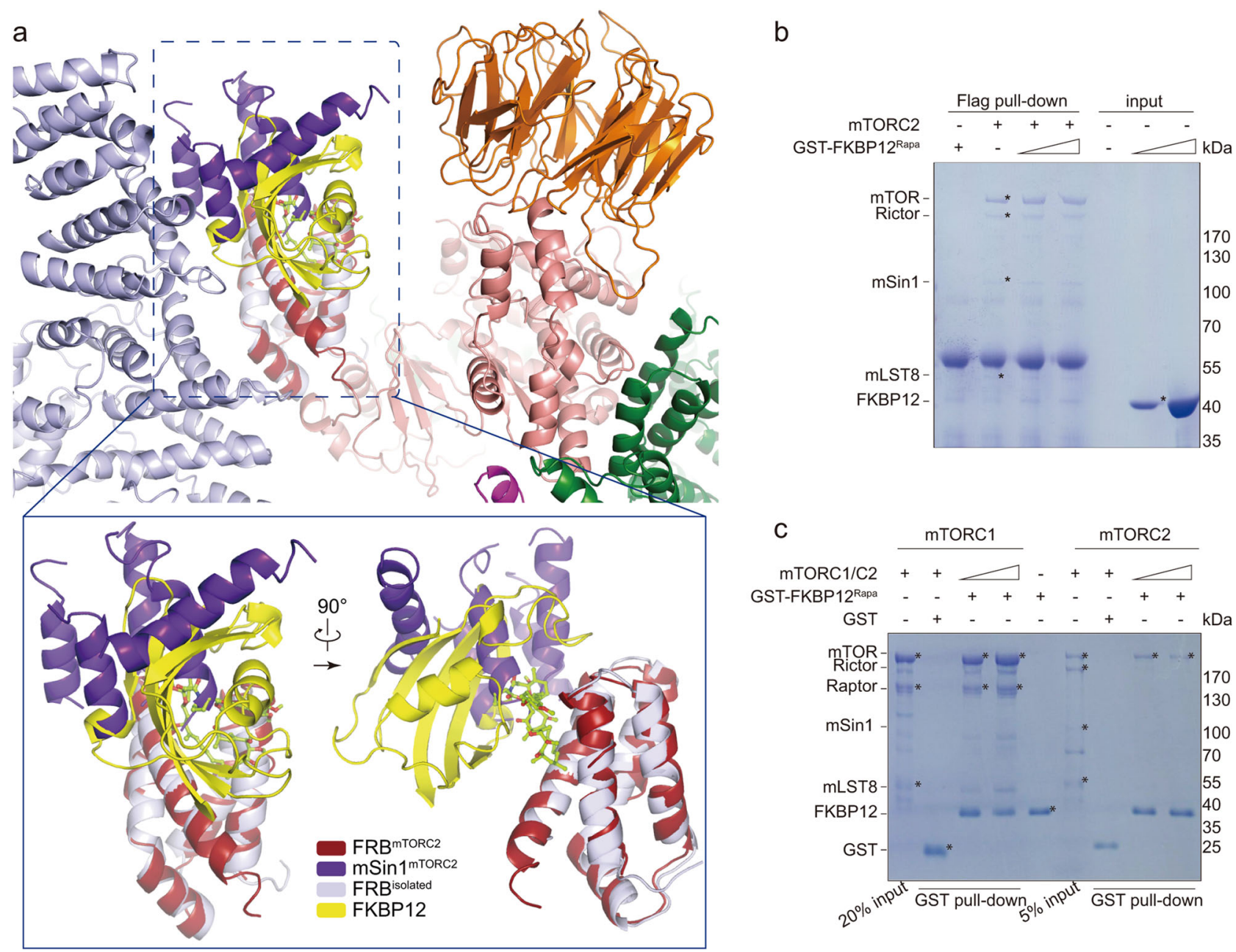

Fig. 7 Mechanism for rapamycin insensitivity of mTORC2. a Superimposition of the mTORC2 and FKBP12-rapamycin-FRB structures shown in ribbon representations (upper panel). Closed-up views of the superimposed structure shown in two different views (lower panel). FKBP12rapamycin is colored in yellow and rapamycin is shown in stick representation. b, c Flag (b) or GST (c) pull-down assays were performed using purified mTORC2, mTORC1, and GST-tagged FKBP12 in the presence of rapamycin. The protein complexes were incubated and immobilized to the indicated resins, and the bound proteins were subjected to SDS-PAGE and stained using Coomassie blue

According to the $4.9 \AA$ EM map, we could only build polyAlanine chains for $\mathrm{N}$-terminal portion of Rictor and five a-helices of $\mathrm{mSin} 1$. The rest portions are invisible in the EM map possibly due to their intrinsic flexibility, which is consistent with secondary structure prediction (Fig. 1a). We observed extra density near the four-helix bundle and the catalytic pocket of mTOR (Supplementary information, Figure S5a lower panel, d). This flattened stretch density might represent the $\beta$-sheet from other domains of $m \operatorname{Sin} 1$. For example, the structure of Schizosaccharomyces pombe $\operatorname{Sin} 1^{\text {CRIM }}$ shows a four-stranded $\beta$-sheet packing against three a-helices. ${ }^{30,31}$ It is tempting to speculate that the flattened stretch density is derived from the CRIM domain, which has been shown to recruit substrate for phosphorylation by TORC2. ${ }^{30,32}$

Previous study shows that the $\mathrm{PH}$ domain of $\mathrm{mSin} 1$ inhibits the kinase activity of $\mathrm{mTORC} 2$ and this autoinhibition is released in the presence of PIP3. ${ }^{20}$ To test whether that is the case, we performed an in vitro kinase assay using purified mTORC2 complexes containing various $C$-terminal truncations of $m \operatorname{Sin} 1$. The $m \operatorname{Sin} 1{ }^{\mathrm{N}}$ containing constructs could form stable mTORC2 complexes (Fig. 2e and Supplementary information, Figure S3c). Intriguingly, we did not detect significant difference in catalytic activity for the complex formed by full-length $\mathrm{mSin} 1$ or truncated $\mathrm{mSin} 1$ lacking $\mathrm{PH}$ domain (Fig. 2e). One possible explanation is that the mTORC2 protein complex was obtained from $293 \mathrm{~F}$ cells cultured in serum- free medium. Under such condition, mTORC2 might adopt an active conformation in which the $\mathrm{PH}$-mediated autoinhibition does not exist for unknown reason.

Previous studies show that the addition of $\mathrm{N}$-terminal tag to $\mathrm{mSin} 1$ or the deletion of $\mathrm{N}$-terminal 192 residues of $\mathrm{mSin} 1$ (mSin1.4, an N-terminal deletion isoform of mSin1.1) largely hampers the mTORC2 complex assembly. ${ }^{15}$ This is consistent with our results and supports that the $\mathrm{N}$-terminal region of $\mathrm{mSin} 1$ is critical for mTORC2 complex assembly, whereas other regions of $\mathrm{mSin} 1$ may play a regulatory role.

When our manuscript was in preparation, Karuppasamy et al. ${ }^{26}$ reported a $7.9 \AA$ cryo-EM structure of S. cerevisiae TORC2 (scTORC2). The EM map of scTORC2 reveals extra density corresponding to the two yeast non-essential components, Avo2 and Bit61. Structural comparison shows that the two complexes adopt similar architectures (Supplementary information, Figure S7). However, the $4.9 \AA$ EM map of human mTORC2 provides a better density for the assignment of Rictor and mSin 1 and structural analyses.

\section{MATERIALS AND METHODS}

Reagents

Antibodies used in this study: anti-phospho-Ser473 AKT (4060, Cell Signaling Technology (CST)), anti-AKT (4691, CST), anti-Rictor 
(2114, CST), anti-mTOR (2972, CST), anti-mSin1 (12860, CST), antiFlag-HRP (A8592, Sigma), anti-myc-HRP (GNI4310-MC-S, GNI GROUP), horseradish peroxidase-labeled anti-rabbit secondary antibodies (AbMart). Other reagents: Flag resin (FLAG M2-agarose, Sigma), rabbit IgG beads (Smart-lifesciences), amylose resin (NEB), rapamycin (Selleck), glutaraldehyde solution (G5882, Sigma), polyethylenimine (PEl; 23966, Polysciences), Pierce C18 spin column (GL Sciences), 293F medium (Sino Biological Inc.); glutathione resin, Superdex $75(10 / 300 \mathrm{GL})$ and Superose-6 Increase (5/150 GL) were from GE Healthcare; $3 \times$ Flag peptide was synthesized in Meilunbio Inc.; Torin 1 was kindly provided by Dr. Haixin Yuan (IBS, Fudan University).

Protein expression and purification

To produce soluble mTORC2 protein complex, the ORFs of human mTOR, Rictor, mSin 1 and $\mathrm{mLST} 8$ were sub-cloned into four different modified pCAG vectors, which contain an N-terminal myc tag (mTOR), N-terminal Flag tag (Rictor), C-terminal ProA tag (mSin1), N-terminal Flag tag (mLST8), respectively. The four plasmids were co-transfected to $293 \mathrm{~F}$ cells using PEl. After cultured at $37^{\circ} \mathrm{C}$ for $72 \mathrm{~h}$, cells were harvested and lysed in lysis buffer (50 mM HEPES, pH 8.0, $300 \mathrm{mM} \mathrm{NaCl}, 0.1 \%$ CHAPS, $5 \mathrm{mM}$ ATP, $5 \mathrm{mM} \mathrm{MgCl} 2,2 \mathrm{mM}$ EDTA, $3 \mathrm{mM}$ DTT, 10\% (v/v) glycerol, 1 $\mathrm{mM}$ PMSF, $1 \mu \mathrm{g} / \mathrm{ml}$ aprotinin, $1 \mu \mathrm{g} / \mathrm{ml}$ pepstatin, $1 \mu \mathrm{g} / \mathrm{ml}$ leupeptin) at $4{ }^{\circ} \mathrm{C}$ for $30 \mathrm{~min}$. The lysates were clarified by centrifugation at 15,000 r.p.m. at $4{ }^{\circ} \mathrm{C}$ for $30 \mathrm{~min}$. Supernatants were mixed with Flag resin and incubated for $1.5 \mathrm{~h}$. The resins were thoroughly washed with the lysis buffer. The fusion proteins were digested using TEV protease at $4{ }^{\circ} \mathrm{C}$ for $2 \mathrm{~h}$ to remove tags and the complex was eluted by an elution buffer ( $50 \mathrm{mM}$ HEPES, pH 8.0, $300 \mathrm{mM}$ $\mathrm{NaCl}, 0.1 \%$ CHAPS, $0.5 \mathrm{mM}$ EDTA, $3 \mathrm{mM}$ DTT, 10\% (v/v) glycerol). The protein complex was concentrated and stored at $-80^{\circ} \mathrm{C}$ for kinase assay and XL-MS analysis. The mTORC2 complexes with mSin 1 truncations were similarly prepared.

To prepare $\mathrm{mTORC2}$ sample for EM study, the mTORC2 proteins were concentrated to $5 \mathrm{mg} / \mathrm{ml}$ followed by gradient fixation (Grafix). ${ }^{33}$ The gradient was generated from a $10 \%$ glycerol light solution (10\% (v/v) glycerol, $300 \mathrm{mM} \mathrm{NaCl}, 30 \mathrm{mM}$ HEPES, pH 7.4, $0.5 \mathrm{mM}$ EDTA, $1 \mathrm{mM}$ TCEP), and a $30 \%$ glycerol heavy solution $(30 \%(\mathrm{v} / \mathrm{v})$ glycerol, $300 \mathrm{mM} \mathrm{NaCl}, 30 \mathrm{mM}$ HEPES, pH 7.4, $0.5 \mathrm{mM}$ EDTA, $1 \mathrm{mM}$ TCEP, and $0.15 \%$ (v/v) glutaraldehyde). Centrifugation was performed at 38,000 r.p.m. in a SW41Ti swinging bucket rotor for $18 \mathrm{~h}$ at $4{ }^{\circ} \mathrm{C}$. Subsequently, peak fractions were collected and quenched with $100 \mathrm{mM}$ Tris- $\mathrm{HCl}$ (pH 8.0). The cross-linked mTORC2 complex was further purified by gel filtration chromatography (Superose-6 Increase 5/150 GL) in the buffer containing $300 \mathrm{mM} \mathrm{NaCl}, 30 \mathrm{mM}$ HEPES (pH 7.4), $0.5 \mathrm{mM}$ EDTA and $1 \mathrm{mM}$ TCEP. The peak fractions were concentrated to $1.5 \mathrm{mg} / \mathrm{ml}$ and applied to Cryo-EM grids.

To prepare Flag-tagged mTORC2 for in vitro pull-down assay, the proteins were directly eluted by an elution buffer $(0.5 \mathrm{mg} / \mathrm{ml}$ $3 \times$ Flag peptide, $50 \mathrm{mM}$ HEPES, pH 7.4, $300 \mathrm{mM} \mathrm{NaCl}, 0.1 \%$ CHAPS, $0.5 \mathrm{mM}$ EDTA, $3 \mathrm{mM}$ DTT) from the Flag resin. The complexes were concentrated and stored at $-80^{\circ} \mathrm{C}$ for the pull-down assay.

The full-length variant of human AKT (K179D, kinase-dead mutant) was sub-cloned into the modified pCAG vectors with an $\mathrm{N}$-terminal ProA tag. The plasmids were transfected into $293 \mathrm{~F}$ cells. The cells were harvested and lysed in lysis buffer $(50 \mathrm{mM}$ HEPES, pH 7.4, $300 \mathrm{mM} \mathrm{NaCl}, 0.25 \%$ CHAPS, $5 \mathrm{mM}$ ATP, $5 \mathrm{mM}$ $\mathrm{MgCl}_{2}, 3 \mathrm{mM}$ DTT, $1 \mathrm{mM}$ PMSF, $1 \mu \mathrm{g} / \mathrm{ml}$ Aprotinin, $1 \mu \mathrm{g} / \mathrm{ml}$ Pepstatin, $1 \mu \mathrm{g} / \mathrm{ml}$ Leupeptin). The clarified lysate was applied to IgG beads and the fusion protein was digested with Precission protease. The protein was further purified by gel filtration chromatography (Superdex 75, 10/300 GL) in the buffer containing $50 \mathrm{mM}$ HEPES (pH 7.4), $150 \mathrm{mM} \mathrm{NaCl}$ and $1 \mathrm{mM}$ TCEP. The peak fractions were collected and stored at $-80^{\circ} \mathrm{C}$ for kinase assays.

GST-FKBP12 protein was expressed in E. coli BL21 (DE3) cells transformed with modified pGEX-6P-1. The fusion protein was purified by glutathione resin and gel filtration chromatography (Superdex 75, 10/300 GL) in the buffer containing $50 \mathrm{mM}$ HEPES $(\mathrm{pH} 7.4), 150 \mathrm{mM} \mathrm{NaCl}$ and $1 \mathrm{mM}$ TCEP. The peak fractions were collected and stored at $-80^{\circ} \mathrm{C}$ for the pull-down assay.

The Flag-tagged human mTORC1 was overexpressed in mammalian $293 \mathrm{~F}$ cells and purified as described previously. ${ }^{23}$ In brief, the full-length mTOR, Raptor and mLST8 were sub-cloned into the three modified pCAG vectors with N-terminal Flag tag fusion in Raptor and mLST8, and co-transfected into 293F cells. The cells were harvested and lysed in lysis buffer (50 mM HEPES, $\mathrm{pH} 7.4,150 \mathrm{mM} \mathrm{NaCl}, 0.4 \%$ CHAPS, $3 \mathrm{mM}$ DTT, $1 \mathrm{mM}$ PMSF, $1 \mu \mathrm{g} /$ $\mathrm{ml}$ Aprotinin, $1 \mu \mathrm{g} / \mathrm{ml}$ Pepstatin, $1 \mu \mathrm{g} / \mathrm{ml}$ Leupeptin). The clarified lysate was applied to Flag resin, and the proteins were eluted by an elution buffer containing $0.5 \mathrm{mg} / \mathrm{ml} 3 \times$ Flag peptide, $50 \mathrm{mM}$ HEPES (pH 7.4), $150 \mathrm{mM} \mathrm{NaCl}$ and $3 \mathrm{mM}$ DTT. The protein was concentrated and stored at $-80^{\circ} \mathrm{C}$ for the pull-down assay.

In vitro kinase assays

The in vitro kinase assays were performed in the reaction buffer containing $25 \mathrm{mM}$ HEPES (pH 7.4), $100 \mathrm{mM} \mathrm{KAC}$, and $1 \mathrm{mM} \mathrm{MgCl}$. The purified human AKT (K179D) serves as a substrate. Purified mTORC2 complex was mixed with AKT (K179D) in a $15 \mu$ reaction. Reactions were initiated by the addition of $0.5 \mathrm{mM} \mathrm{ATP}$, and incubated for $30 \mathrm{~min}$ at $37^{\circ} \mathrm{C}$. Reactions were terminated by the addition of SDS-PAGE sample loading buffer and analyzed by western blotting.

In vitro pull-down assay

The purified GST-FKBP12 was incubated with rapamycin for 20 min on ice in equal molar ratio before pull-down assay. In the GST pull-down assay, $150 \mathrm{nM}$ pre-incubated GST-FKBP12-rapamycin was incubated with the purified Flag-tagged mTORC1 complex $(30 \mathrm{nM}, 75 \mathrm{nM})$ and Flag-tagged mTORC2 complex (30 nM, $75 \mathrm{nM})$ in $300 \mu \mathrm{l}$ of pull-down buffer ( $30 \mathrm{mM}$ HEPES, pH 7.4, $150 \mathrm{mM} \mathrm{NaCl}$, $0.1 \%$ CHAPS, $0.5 \mathrm{mM}$ EDTA, $3 \mathrm{mM}$ DTT) for $15 \mathrm{~min}$ on ice, respectively. Glutathione resins were washed four times with the pull-down buffer and then mixed with the proteins at $4^{\circ} \mathrm{C}$ for $1 \mathrm{~h}$. After being washed three times with the pull-down buffer, the immobilized proteins were subjected to SDS-PAGE and stained by Coomassie blue.

In the Flag pull-down assay, $15 \mathrm{nM}$ purified Flag-tagged mTORC2 protein was incubated with increasing amount of preincubated GST-FKBP12-rapamycin (75 nM, $150 \mathrm{nM}, 750 \mathrm{nM}, 1.5$ $\mu M)$ in $300 \mu$ of pull-down buffer for $15 \mathrm{~min}$ on ice, respectively. Flag resins were washed four times with the pull-down buffer and then mixed with the proteins at $4{ }^{\circ} \mathrm{C}$ for $1.5 \mathrm{~h}$. After being washed three times with the pull-down buffer, the bound proteins were subjected to SDS-PAGE and stained by Coomassie blue.

\section{Co-immunoprecipitation assays}

The indicated plasmids were co-transfected to $10 \mathrm{ml}$ of $293 \mathrm{~F}$ cells and cultured at $37^{\circ} \mathrm{C}$ for $72 \mathrm{~h}$. The cells were harvested and lysed in lysis buffer containing $50 \mathrm{mM}$ HEPES (pH 8.0), $300 \mathrm{mM} \mathrm{NaCl}$, $0.1 \%$ CHAPS, $5 \mathrm{mM}$ ATP, $5 \mathrm{mM} \mathrm{MgCl} 2,2 \mathrm{mM}$ EDTA, $3 \mathrm{mM}$ DTT, $10 \%$ (v/v) glycerol, $1 \mathrm{mM}$ PMSF, $1 \mu \mathrm{g} / \mathrm{ml}$ aprotinin, $1 \mu \mathrm{g} / \mathrm{ml}$ pepstatin, 1 $\mu \mathrm{g} / \mathrm{ml}$ leupeptin. Supernatants were incubated with the indicated resins at $4^{\circ} \mathrm{C}$ for $1.5 \mathrm{~h}$. After being washed three times with wash buffer ( $50 \mathrm{mM}$ HEPES, pH 7.4, $300 \mathrm{mM} \mathrm{NaCl}, 5 \mathrm{mM} \mathrm{MgCl}, 5 \mathrm{mM}$ ATP, $0.1 \%$ CHAPS, $3 \mathrm{mM}$ DTT, $10 \%$ glycerol), the immobilized proteins were subjected to SDS-PAGE and visualized by Commassie blue staining and/or western blotting using the indicated antibodies.

Cross-linking and mass spectrometry analysis

The purified mTORC2 $(1.2 \mu \mathrm{g} / \mu \mathrm{l})$ was cross-linked with disuccinimidyl suberate (DSS) in a 1:150 molar ratio at room temperature for $20 \mathrm{~min}$. The reaction was quenched by adding $20 \mathrm{mM}$ ammonium bicarbonate. Cross-linked sample was digested with 
trypsin overnight. After being quenched by $5 \%$ formic acid, the tryptic peptides were desalted with Pierce C18 spin column and separated in a proxeon EASY-nLC liquid chromatography system by applying a step-wise gradient of $0 \%-85 \%$ acetonitrile (ACN) in $0.1 \%$ formic acid. Peptides eluted from the LC column were directly electrosprayed into the mass spectrometer with a distal 2 kV spray voltage. Data-dependent tandem mass spectrometry (MS/MS) analysis was performed on Thermo Q-Exactive instrument in a 60-min gradient. Raw data was processed with pLink software, ${ }^{34}$ and the results were visualized using the xiNET online server (Rappsilber Laboratory, University of Edinburgh, Scotland; crosslinkviewer.org). ${ }^{35}$

\section{EM data acquisition}

For negative staining EM, $5 \mu \mathrm{l}$ of mTORC2 complex was applied to glow-discharged copper grids supported by a thin layer of carbon film for 1 min before negative staining by uranyl formate $(2 \%, w / v)$ solution at room temperature. The negatively stained grid was loaded to FEI Talos L120C operated at $120 \mathrm{kV}$ for evaluation of the protein quality.

For cryo-EM grid preparation, aliquots of $4 \mu \mathrm{l}$ of mTORC2 complex $(\sim 1.5 \mathrm{mg} / \mathrm{ml})$ were applied to glow-discharged holey carbon grids (Quantifoil Au, R1.2/1.3, 400 mesh). The grids were then blotted for 3 or $5 \mathrm{~s}$ and flash-plunged into liquid ethane precooled by liquid nitrogen using an FEI Vitrobot mark IV operated at $10{ }^{\circ} \mathrm{C}$ and $100 \%$ humidity. Cryo-EM grids were loaded onto an FEl Titan Krios microscope equipped with K2 Summit camera for data collection. All the cryo-EM images were automatically recorded at counting mode using Serial-EM. ${ }^{36}$ The magnification is 29,000 , corresponding to a pixel size of $1.01 \AA$. For each image stack, a total dose of about 50 electrons were equally fractioned into 32 frames with a total exposure time of $8 \mathrm{~s}$. Defocus values used to collect the dataset ranged from -1.7 to $-4.4 \mu \mathrm{m}$.

\section{Image processing}

For cryo-EM data, beam-induced motion correction was performed using the MotionCor2 to generate average micrographs and dose-weighted micrographs from all frames. ${ }^{37}$ The contrast transfer function parameters were estimated by CTFFIND $4^{38}$ from averaged micrographs. Other procedures of cryo-EM data processing were performed with RELION 1.4 or RELION 2.0 using the dose-weighted micrographs. ${ }^{39}$ After two rounds of reference-free 2D classification, 294,995 particles were then subjected to 3D classification with the previously reconstructed mTORC1 cryo-EM map (EMDB: 6668) as the initial model after low-pass filtered to 60 $\AA$. Then, 195,353 particles were used for refinement with the imposition of C2 symmetry. A reported $4.92 \AA$ resolution (corrected gold-standard FSC 0.143 criteria) map was generated after B-factor sharpening with a B-factor of $-244 \AA^{2}$ (Postprocessing in RELION). ${ }^{36}$ All the visualization and evaluation of the $3 \mathrm{D}$ volume map was performed using Chimera, ${ }^{40}$ and the local resolution map was calculated using ResMap. ${ }^{41}$ The procedures for data collection are summarized in Supplementary information, Figure S4.

Model building into the cryo-EM map

The cryo-EM structure of mTOR/mLST8 (PDB ID: $6 B C X)^{24}$ was docked into the mTORC2 cryo-EM map using EMfit ${ }^{42}$ while maintaining the two-fold symmetry. The models of Rictor and mSin 1 were manually built using COOT. ${ }^{43}$ The coordinates of the final structure were refined in the real space using phenix. real_space_refine. ${ }^{44}$ Model validation was performed with PRO$\mathrm{CHECK}^{45}$ and the WHATCHECK routine of WHAT IF. ${ }^{46}$

Accession codes

The electron density map and corresponding atomic coordinates have been deposited in the Protein Data Bank (http://www.rcsb. org/pdb) with code: 5ZCS and EMDB (http://www.ebi.ac.uk/pdbe/ emdb/) with code: EMD-6913.

\section{ACKNOWLEDGEMENTS}

We thank Center of Cryo-Electron Microscopy, Zhejiang University School of Medicine, Center for Biological Imaging of Institute of Biophysics (IBP) of Chinese Academy of Sciences (CAS), and National Center for Protein Science Shanghai (NCPSS) for the support on cryo-EM data collection and analyses. We thank the staff members at Biomedical Core Facility, Fudan University and NCPSS for their help with mass spectrometry analyses. This work was supported by the Ministry of Science and Technology of China (2016YFA0500700, 2016YFA0501100), the National Natural Science Foundation of China (31770781, U1432242, 31425008, 91419301), the National Program for support of Top-Notch Young Professionals (Y.X.), and the Strategic Priority Research Program of CAS (XDB08000000).

\section{AUTHOR CONTRIBUTIONS}

X.C., M.L., H.Y., and Y.X. designed the experiments. X.C., J.L., and D.Z. purified the proteins. M.L., Y.T., Y.Q., H-W.W., and Z.W. prepared the cryo-EM sample, collected the data and determined the structure. J.W. built the structural model. X.C. and H.Y. performed biochemical analyses. X.C., M.H., and C.L. performed cross-linking MS and analyzed the data. X.C., M.L., H.Y., and Y.X. analyzed the data and wrote the manuscript. Y.X. supervised the project.

\section{ADDITIONAL INFORMATION}

Supplementary information accompanies this paper at https://doi.org/10.1038/ s41422-018-0029-3.

Competing interests: The authors declare no competing interests.

\section{REFERENCES}

1. Heitman, J., Movva, N. R. \& Hall, M. N. Targets for cell cycle arrest by the immunosuppressant rapamycin in yeast. Science 253, 905-909 (1991).

2. Sabatini, D. M., Erdjument-Bromage, H., Lui, M., Tempst, P. \& Snyder, S. H. RAFT1: a mammalian protein that binds to FKBP12 in a rapamycin-dependent fashion and is homologous to yeast TORs. Cell 78, 35-43 (1994).

3. Loewith, R. et al. Two TOR complexes, only one of which is rapamycin sensitive, have distinct roles in cell growth control. Mol. Cell 10, 457-468 (2002).

4. Wedaman, K. P. et al. Tor kinases are in distinct membrane-associated protein complexes in Saccharomyces cerevisiae. Mol. Biol. Cell 14, 1204-1220 (2003).

5. Saxton, R. A. \& Sabatini, D. M. mTOR signaling in growth, metabolism, and disease. Cell 168, 960-976 (2017).

6. Wullschleger, S., Loewith, R. \& Hall, M. N. TOR signaling in growth and metabolism. Cell 124, 471-484 (2006).

7. Sarbassov, D. D., Guertin, D. A., Ali, S. M. \& Sabatini, D. M. Phosphorylation and regulation of Akt/PKB by the rictor-mTOR complex. Science 307, 1098-1101 (2005).

8. Sarbassov, D. D. et al. Rictor, a novel binding partner of mTOR, defines a rapamycin-insensitive and raptor-independent pathway that regulates the cytoskeleton. Curr. Biol. 14, 1296-1302 (2004).

9. Garcia-Martinez, J. M. \& Alessi, D. R. mTOR complex 2 (mTORC2) controls hydrophobic motif phosphorylation and activation of serum- and glucocorticoidinduced protein kinase 1 (SGK1). Biochem. J. 416, 375-385 (2008).

10. Sparks, C. A. \& Guertin, D. A. Targeting mTOR: prospects for mTOR complex 2 inhibitors in cancer therapy. Oncogene 29, 3733-3744 (2010).

11. Kim, D. H. et al. GbetaL, a positive regulator of the rapamycin-sensitive pathway required for the nutrient-sensitive interaction between raptor and mTOR. Mol. Cell 11, 895-904 (2003).

12. Kim, D. H. et al. mTOR interacts with raptor to form a nutrient-sensitive complex that signals to the cell growth machinery. Cell 110, 163-175 (2002).

13. Hara, K. et al. Raptor, a binding partner of target of rapamycin (TOR), mediates TOR action. Cell 110, 177-189 (2002).

14. Jacinto, E. et al. Mammalian TOR complex 2 controls the actin cytoskeleton and is rapamycin insensitive. Nat. Cell Biol. 6, 1122-1128 (2004).

15. Frias, M. A. et al. $m S i n 1$ is necessary for Akt/PKB phosphorylation, and its isoforms define three distinct mTORC2s. Curr. Biol. 16, 1865-1870 (2006).

16. Jacinto, E. et al. SIN1/MIP1 maintains rictor-mTOR complex integrity and regulates Akt phosphorylation and substrate specificity. Cell 127, 125-137 (2006).

17. Baretic, D. \& Williams, R. L. PIKKs - the solenoid nest where partners and kinases meet. Curr. Opin. Struct. Biol. 29, 134-142 (2014). 
18. Keith, C. T. \& Schreiber, S. L. PIK-related kinases: DNA repair, recombination, and cell cycle checkpoints. Science 270, 50-51 (1995).

19. Yang, $H$. et al. mTOR kinase structure, mechanism and regulation. Nature 497, 217-223 (2013).

20. Liu, P. et al. Ptdlns $(3,4,5) P 3-d e p e n d e n t$ activation of the mTORC2 kinase complex. Cancer Discov. 5, 1194-1209 (2015).

21. Aylett, C. H. et al. Architecture of human mTOR complex 1. Science 351, 48-52 (2016).

22. Baretic, D., Berndt, A., Ohashi, Y., Johnson, C. M. \& Williams, R. L. Tor forms a dimer through an $\mathrm{N}$-terminal helical solenoid with a complex topology. Nat. Commun. 7, 11016 (2016)

23. Yang, H. et al. 4.4 A Resolution cryo-EM structure of human mTOR complex 1. Protein Cell 7, 878-887 (2016).

24. Yang, $H$. et al. Mechanisms of mTORC1 activation by RHEB and inhibition by PRAS40. Nature 552, 368-373 (2017).

25. Gaubitz, C. et al. Molecular basis of the rapamycin insensitivity of target of rapamycin complex 2. Mol. Cell 58, 977-988 (2015).

26. Karuppasamy, M. et al. Cryo-EM structure of Saccharomyces cerevisiae target of rapamycin complex 2. Nat. Commun. 8, 1729 (2017).

27. Gaubitz, C., Prouteau, M., Kusmider, B. \& Loewith, R. TORC2 structure and function. Trends Biochem. Sci. 41, 532-545 (2016).

28. Zhou, P., Zhang, N., Nussinov, R. \& Ma, B. Defining the domain arrangement of the mammalian target of rapamycin complex component Rictor protein. J. Comput. Biol. 22, 876-886 (2015).

29. Choi, J., Chen, J., Schreiber, S. L. \& Clardy, J. Structure of the FKBP12-rapamycin complex interacting with the binding domain of human FRAP. Science 273, 239-242 (1996).

30. Tatebe, $\mathrm{H}$. et al. Substrate specificity of TOR complex 2 is determined by a ubiquitin-fold domain of the $\operatorname{Sin} 1$ subunit. Elife 6, https://doi.org/10.7554/ eLife.19594 (2017).

31. Furuita, K. et al. Utilization of paramagnetic relaxation enhancements for highresolution NMR structure determination of a soluble loop-rich protein with sparse NOE distance restraints. J. Biomol. NMR 61, 55-64 (2015).
32. Cameron, A. J., Linch, M. D., Saurin, A. T., Escribano, C. \& Parker, P. J. mTORC2 targets AGC kinases through Sin1-dependent recruitment. Biochem. J. 439, 287-297 (2011).

33. Kastner, B. et al. GraFix: sample preparation for single-particle electron cryomicroscopy. Nat. Methods 5, 53-55 (2008).

34. Yang, B. et al. Identification of cross-linked peptides from complex samples. Nat. Methods 9, 904-906 (2012).

35. Combe, C. W., Fischer, L. \& Rappsilber, J. xiNET: cross-link network maps with residue resolution. Mol. Cell Proteom. 14, 1137-1147 (2015).

36. Mastronarde, D. N. Automated electron microscope tomography using robust prediction of specimen movements. J. Struct. Biol. 152, 36-51 (2005).

37. Zheng, S. Q. et al. MotionCor2: anisotropic correction of beam-induced motion for improved cryo-electron microscopy. Nat. Methods 14, 331-332 (2017).

38. Rohou, A. \& Grigorieff, N. CTFFIND4: Fast and accurate defocus estimation from electron micrographs. J. Struct. Biol. 192, 216-221 (2015).

39. Scheres, S. H. RELION: implementation of a Bayesian approach to cryo-EM structure determination. J. Struct. Biol. 180, 519-530 (2012).

40. Pettersen, E. F. et al. UCSF Chimera - a visualization system for exploratory research and analysis. J. Comput. Chem. 25, 1605-1612 (2004).

41. Kucukelbir, A., Sigworth, F. J. \& Tagare, H. D. Quantifying the local resolution of cryo-EM density maps. Nat. Methods 11, 63-65 (2014).

42. Rossmann, M. G., Bernal, R. \& Pletnev, S. V. Combining electron microscopic with X-ray crystallographic structures. J. Struct. Biol. 136, 190-200 (2001).

43. Emsley, P., Lohkamp, B., Scott, W. \& Cowtan, K. Features and development of Coot. Acta Crystallogr. D Biol. Crystallogr. 66, 486-501 (2010).

44. Adams, P. D. et al PHENIX: a comprehensive Python-based system for macromolecular structure solution. Acta Crystallogr. D Biol. Crystallogr. 66, 213-221 (2010).

45. Laskowski, R. A., MacArthur, M. W., Moss, D. S. \& Thornton, J. M. PROCHECK: a program to check the stereochemical quality of protein structures. J. Appl. Crystallogr 26, 283-291 (1993).

46. Vriend, G. WHAT IF: a molecular modeling and drug design program. J. Mol. Graph. 8, 52-56 (1990). 